\title{
Associação entre os indicadores antropométricos e a gordura corporal relativa em homens estudantes da Universidade Federal do Amapá, AP
}

\author{
Association between the anthropometric indicators and percentage \\ body fat in male students of the Federal University of Amapá, AP
}

\section{Wollner Materko ${ }^{1}$ \\ Paulo Jorge de Abreu da Costa Junior ${ }^{2}$}

\footnotetext{
1 Doutorado e Mestrado em Ciências da Saúde em Engenharia Biomédica pelo Instituto Alberto Luiz Coimbra de Pós-graduação e Pesquisa de Engenharia da Universidade Federal do Rio de Janeiro. Especialista em Docência no Ensino Superior pela Universidade Cândido Mendes. graduado em Educação Física pela Universidade Estácio de Sá - UNESA/RJ. Professor adjunto da Universidade Federal do Amapá

E-mail: wollner.materko@gmail.com 19 http://lattes.cnpq.br/7169355551481923 (D) https://orcid.org/0000-0002-9000-0201 2 Estudante da Universidade Federal do Amapá.

RESUMO: A obesidade é definida pela Organização Mundial da Saúde (OMS) como uma doença caracterizada pelo acúmulo de gordura corporal, sendo de grande importância desenvolver pontos de corte baseado em indicadores antropométricos para o diagnóstico da obesidade como medida de prevenção e intervenção na saúde pública. Sendo assim, o objetivo do presente estudo verificou a relação dos indicadores antropométricos com a gordura corporal relativa em homens estudantes do curso de graduação em Educação Física da Universidade Federal do Amapá. Participaram deste estudo uma amostra de 22 homens (20 a 29 anos) aparentemente saudável. A seleção da amostragem foi feita por conglomerados, pois os voluntários foram selecionados aleatoriamente durante uma avaliação antropométrica para as medida da circunferência da cintura (CC), da circunferência do abdômen (CA), da circunferência do quadril (CQ), a razão cintura e quadril (RCQ) e do índice de massa corporal (IMC) e a gordura corporal relativa (GCR) pelo método por dobras cutâneas. O presente estudo demonstrou uma boa relação linear e significativa $(r=0,50-0,75)$ entre os indicadores antropométricos e a GCR. Em conclusão, foram observadas correlações significantes entre todos os indicadores antropométricos e a GCR quando analisados em voluntários com características físicas e antropométricas similares às do presente estudo, tornando-se possíveis indicadores na predição de doenças e na promoção de saúde.

Palavras-chave: Composição corporal. Índice de massa corporal. Circunferência abdominal. Razão cintura e quadril.

\begin{abstract}
Obesity is defined by the World Health Organization (WHO) as a disease characterized by the excessive accumulation of body fat, being of great importance to develop cut-off points based on anthropometric indicators for the diagnosis of obesity as a preventive measure and intervention in public health. This study aimed at determining the association between anthropometric indicators and body fat percentage in male students of Physical Education course at the Federal University of Amapá. A sample of 22 apparently healthy men (20-29 years) participated in this study. Sampling was performed by clusters, since the volunteers were randomly selected during an anthropometric evaluation for waist circumference, abdomen circumference, hip circumference, waist-hip ratio, body mass index and percentage body fat $(\% \mathrm{BF})$ by the skinfold method. The present study showed a good linear correlated and significant $(r=0.50-0.75)$ between the anthropometric and $\% \mathrm{BF}$. In conclusion, it resulted significant correlations between all of the anthropometric indicators and $\% \mathrm{BF}$ when analyzed in volunteers with physical and anthropometric characteristics similar to those of the present study, becoming possible indicators in the prediction of diseases and health promotion.
\end{abstract}

Keywords: Body composition. Body mass index. Abdomen circumference. Waist-hip ratio. 


\section{INTRODUÇÃO}

Obesidade é definida pela Organização Mundial da Saúde (OMS) como uma doença caracterizada pelo acúmulo excessivo de gordura corporal, além de ser considerada um problema de saúde pública que leva a sérias consequências sociais, psicológicas e físicas, sendo associada a um maior risco de morbimortalidade por doenças crônicas não transmissíveis (WORLD HEALTH ORGANIZATION, 2000). Tem sido observada forte associação entre a obesidade e dislipidemia (MANGGE et al., 2010), hipertensão arterial (SARNO; MONTEIRO, 2007) e hiperinsulimia (CASTRO; MATO; GOMES, 2006).

A prevalência de sobrepeso e obesidade vem aumentando em vários países, inclusive no Brasil, onde o sobrepeso passou de 51,1\% em 2010, para 54,1 em 2014 (BRASIL, 2016). Em 2010, 17,8\% da população brasileira era obesa; em 2014, o índice chegou aos 20\%, sendo a maior prevalência entre as mulheres, $22,7 \%$, deixando de ser uma preocupação meramente estética para se transformar num problema grave de saúde pública (BRASIL, 2016).

Em estudos epidemiológicos é amplamente conhecida a relação entre a deposição de gordura na região abdominal e enfermidades, pois é altamente correlacionada com diversas doenças crônicas, como cardiopatia coronária, hipertensão arterial, diabetes tipo II e aumento no risco de mortalidade (GRINKER, 2000; OKOSUN et al., 2000). A obesidade central é associada com o aumento do tecido adiposo visceral (CLASEY et al., 1999; SCHREINER et al., 1996; POULIO'T et al., 1994), e está independentemente associada com o nível de glicose e na concentração de insulina para ambos os sexos (GARAULET et al., 2000; KARTER et al., 1996; FUJIOKA et al., 1987).

Diante da gravidade do problema da obesidade e, ainda, pelo frequente insucesso em que resulta a maioria dos tratamentos, poderia ser mais fácil, barato e efetivo em desenvolver pontos de corte baseado em indicadores antropométricos para o diagnóstico da obesidade, em relação a gordura corporal relativa (MATERKO et al., 2017), como medida de prevenção, intervenção na saúde pública e na promoção de saúde. Sendo assim, o objetivo do estudo foi de verificar a relação dos indicadores antropométricos com a gordura corporal relativa em homens estudantes do curso de graduação em Educação Física da Universidade Federal do Amapá situado noroeste da Região do Norte do Brasil, denominado também como Região Amazônica.

\section{MATERIAIS E MÉTODOS}

\subsection{Tipo de estudo e casuística}

Participaram deste estudo uma amostra de 22 homens (20 a 29 anos) e aparentemente saudável seguindo os critérios de classificação do American College Sports Medicine (2014). A seleção da amostragem foi feita por conglomerados, pois os voluntários foram selecionados aleatoriamente durante uma avaliação antropométrica no curso de Educação Física na Universidade Federal do Amapá (UNIFAP) entre outubro a novembro de 2017 no período de 10:00 às 12:00 horas.

Os procedimentos experimentais tiveram início somente após o consentimento verbal e à assinatura do termo de consentimento livre e esclarecido, conforme aprovado pelo Comitê de Ética em Pesquisa da Instituição (CAAE: 57038316.9.0000.0003, Parecer 1.738.671), de acordo com a Resolução 466/2012 do Conselho Nacional de Saúde.

Consideraram-se como critérios de inclusão: sem uso de recursos ergorgênicos, sem lesões osteomioarticulares prévias e sem a utilização de prótese ou marcapasso cardíaco. Todos foram previamente instruídos a não realizar exercício físico nas $24 \mathrm{~h}$ precedentes, 
não consumir bebida alcoólica nas $12 \mathrm{~h}$ precedentes e a manter-se hidratados.

Medidas antropométricas

A coleta da medida da massa corporal e estatura por meio de uma balança com estadiômetro (Filizola, Brasil) com capacidade máxima de $150 \mathrm{Kg}$ e variação de $0,5 \mathrm{Kg}$ para massa corporal e uma variação de $0,1 \mathrm{~cm}$ para estatura, com os voluntários descalços, usando roupas leves e sem portar qualquer objeto. Calculou-se o índice de massa corporal pela razão entre a massa corporal em quilogramas e a estatura do indivíduo em metros (WORLD HEALTH ORGANIZATION, 2000).

circunferências de cintura (CC), abdômen (CA) e quadril (CQ), com os voluntários descalços, usando roupas leves e sem portar qualquer objeto, as medidas das circunferências foram realizadas com uma fita métrica (Cescorf, Brasil), com resolução de $0,1 \mathrm{~cm}$.

Os procedimentos de localização, posicionamento e aferição das circunferências antropométricas foram padronizados de acordo com a International Standards for Anthropometric Assessment (STEWART et al., 2011). A circunferência abdominal (CA) foi obtida na menor curvatura localizada entre as costelas e a crista ilíaca com fita métrica flexível e inelástica sem comprimir os tecidos. Para realização das medidas de relação da cintura/quadril (RCQ), as medidas de circunferências foram realizadas na cintura, e no quadril, sendo que, para circunferência da cintura (CC) os sujeitos permaneceram na posição ortostática, com o abdômen relaxado, a fita foi posicionada menor curvatura localizada entre as costelas e a crista ilíaca com fita métrica flexível e inelástica sem comprimir os tecidos. Para a circunferência do quadril (CQ), os sujeitos continuaram na posição ortostática, sendo a fita posicionada no plano horizontal, ao nível do ponto de maior circunferência da região glútea.

Medidas de dobras cutâneas
As medidas de dobras cutâneas (DC) constaram do pinçamento com o dedo indicador e com o polegar, sempre no lado direito do avaliado, com o compasso entrando perpendicular à dobra; esperou-se de dois segundos a quatro segundos para realizar-se a leitura; o avaliado foi orientado a estar sempre com a pele seca, para que o pinçamento seja possível. Cada DC foi aferida três vezes alternando, pois ao ser aplicado pressão na dobra a gordura subcutânea tende a se comprimir e, assim, diminuir o valor. Se o valor entre as medidas variou acima de um milímetro foi realizada uma nova medida, onde o valor final foi a média entre as três ou quatro medidas (STEWART et al., 2011).

Gordura Corporal Relativa

Para o cálculo da gordura corporal relativa foram aferidas as três DC para homens (peitoral, abdômen e coxa), através de um compasso científico (Cescorf, Brasil). A partir destas medidas, estimou-se a densidade corporal usando as equações de Jackson e Pollock (1978) em homens, conforme as equações 1 e 2 , respectivamente, e, combinada com a equação de Siri (1961) para a estimativa da gordura corporal relativa, como apresentada na equação 2.

$D C_{\text {Homens }}=1,10938-0,0008267 *(X 1)+$ $0,0000016 *(X 1)^{2}-0,00002574 * I$

onde $X 1$ é o somatório das dobras cutâneas do peitoral, abdômen e coxa e I é a idade.

$\left[\% \mathrm{GC}=\left(\frac{495}{D C}\right)-450\right]$

\subsection{Análise Estatística}

Para determinar a normalidade da distribuição, utilizou-se o teste Kolmogorov-Smirnov, verificando-se que a amostra seguiu uma distribuição gaussiana. A análise estatística descritiva buscou a definição do perfil do grupo, sendo expressa como média e desvio padrão, 
além do intervalo de confiança de 95\% (IC95\%). Posteriormente, a relação entre todos os indicadores antropométricos (CA, CQ, CC, RCQ e IMC) e a gordura corporal relativa foi analisada pelo teste de correlação de Pearson. Todos os procedimentos estatísticos foram assumidos como $\alpha=0,05$ para significância estatística e foram processados no software R versão 2.15.0.

\section{RESULTADOS E DISCUSSÕES}

No presente estudo verficou-se a relação dos indicadores antropométricos com a gordura corporal relativa em homens estudantes do curso de graduação em Educação Física da Universidade Federal do Amapá, portanto, baseado nos resultados do presente estudo foi possível verificar uma forte associação linear entre os indicadores antropométricos e a gordura corporal relativa.

A Tabela 1 apresenta as características físicas e antropométricas do grupo de voluntários. A baixa dispersão dos dados devido aos baixos valores de desvio padrão aponta para um grupo bastante homogêneo, confirmando a normalidade da distribuição ao observar o valor p para cada variável.

Os valores de correlação entre os indicadores antropométricos e a gordura corporal relativa estão resumidos na Tabela 2 , mostrando boas associações lineares e significativas.

Têm sido mostradas altas correlações entre a gordura corporal relativa e as variáveis antropométricas (PONGCHAIYAKUL et al., 2005; LEAN; HAN; DEURENBERG, 1996; DEURENBERG; WESTSTRATE; SEIDELL, 1991), de forma que o poder de predição da gordura corporal relativa a partir dessas variáveis tende a ser muito alto (MATERKO; SANTOS, 2010). No presente estudo, só a CA resultou em moderada $(r=0,73)$ correlação com a gordura corporal relativa, similar ao estudo de Grossl, De Lima e Karasiak (2010), que resultou na CA maior correlação com a gordura corporal relativa $(\mathrm{r}=0,73)$ quando comparado aos outros indicadores antropométricos para o sexo masculino.

Tabela 1. Características antropométricas e físicas dos voluntarios Table 1. Anthropometric and physical characteristics of volunteers

\begin{tabular}{llll}
\hline Variáveis & Média \pm DP & $95 \%$ IC & Valor $\mathbf{p}$ \\
\hline Idade (anos) & $23,4 \pm 2,4$ & $22,3-24,5$ & 0,54 \\
Estatura $(\mathrm{cm})$ & $175,4 \pm 6,1$ & $172,6-178,2$ & 0,67 \\
Massa corporal $(\mathrm{kg})$ & $79,3 \pm 12,4$ & $73,6-85,0$ & 0,78 \\
IMC (kg/m²) & $25,9 \pm 4,0$ & $24,0-27,7$ & 0,48 \\
Gordura corporal relativa $(\%)$ & $12,0 \pm 5,1$ & $9,6-14,3$ & 0,14 \\
Circunferência da cintura $(\mathrm{cm})$ & $83,0 \pm 6,7$ & $80,6-86,1$ & 0,96 \\
Circunferência do abdômen $(\mathrm{cm})$ & $86,5 \pm 9,1$ & $82,3-90,6$ & 0,26 \\
Circunferência do quadril $(\mathrm{cm})$ & $100,9 \pm 7,4$ & $97,5-104,3$ & 0,99 \\
RCQ & $0,85 \pm 0,04$ & $0,83-0,87$ & 0,92 \\
\hline \multicolumn{2}{c}{ DP é o desvio padrão e 95\% IC é o intervalo de confiança de $95 \%$}
\end{tabular}

Tabela 2. Correlação entre os indicadores antropométricos e a gordura corporal relativa

Table 2. Correlation between anthropometric indicators and relative body fat

\begin{tabular}{lll}
\hline Indicadores Antropométricos & $\mathbf{R}$ & Valor $\mathbf{p}$ \\
\hline IMC & 0,70 & $<0,01$ \\
Circunferência da cintura & 0,59 & $<0,01$ \\
Circunferência do abdomen & 0,73 & $<0,01$ \\
Circunferência do quadril & 0,50 & 0,01 \\
RCQ & 0,75 & $<0,01$
\end{tabular}

Já a CC é uma variável bastante estudada para avaliar o risco coronariano (LEE et al., 2006), além de apresentar boa correlação ( $\mathrm{r}=$ 0,81-0,88) com a gordura corporal relativa (DEURENBERG; WESTSTRATE; SEIDELL, 1991). Entretanto, o resultado do presente estudo encontrou uma correlação fraca $(\mathrm{r}=0,59)$ com a gordura corporal relativa. Assim como, a variável da CQ resultou em correlação mais fraca, contribuindo $r=0,50$, esse resultado difere dos dados de estudo anterior, realizado com as características antropométricas de mulheres idosas, onde a $\mathrm{CQ}$ resultaram em $\mathrm{r}=0,72$ com a gordura corporal relativa (GONÇALVES, 2004). Pode-se observar que o fraco poder na correlação das variáveis CC e CQ com a gordura corporal relativa para a amostra do presente estudo resultou pelo fato da similaridade da amostra, pois foram constituídos por jovens homens que apresentam um comportamento andróide na distribuição de gordura (MARTI et al., 2004). 
Dividindo a CC pela CQ calcula-se o RCQ que é um indicador antropométrico para avaliação do risco cardiovascular (DA COSTA et al., 2017 ) apresentando uma boa correlação (r $=0,82) \mathrm{com}$ a gordura corporal relativa (OLIVEIRA et al., 2010), similar ao resultado do presente estudo $(r=0,75)$, sendo a variável que apresentou a melhor correlação com a gordura corporal relativa.

Atualmente, o índice de massa corporal (IMC) é expresso como a razão entre a massa corporal em quilogramas e a estatura do indivíduo em metros (KEYS et al., 2014), constitui a ferramenta mais utilizada para o diagnóstico quantitativo da obesidade, devido a sua simplicidade e boa correlação (GROSSL; DE LIMA; KARASIAK, 2010) com a adiposidade corporal, confirmando o resultado do presente estudo $(\mathrm{r}=0,70)$, que mostrou uma boa relação entre o IMC e a gordura corporal relativa, fazendo com que reafirme este indicador como um dos mais fáceis e práticos indicadores antropométricos na predição da gordura corporal relativa (MATERKO; SANTOS, 2010; DEURENBERG; WESTSTRATE; SEIDELL, 1991) e sendo um bom indicativo de ponto de corte baseado na gordura corporal relativa na classificação de indivíduos obesos (MATERKO et al., 2017; MATERKO; SANTOS, 2017).

Cabe apontar que o presente estudo se restringe à população não obesa, pois a coleta de dados foi realizada em estudantes do curso de Educação Física. Recomenda-se o desenvolvimento de trabalhos similares ao presente estudo, para ambos os sexos, , diferentes faixas etárias e, além disso, parece consenso, atualmente, da necessidade de desenvolver pontos de corte dos indicadores antropométricos em função de medidas de composição corporal, tais como, na predição de doenças em amostras da população em estudo como medida de prevenção de Saúde Pública (REZENDE et al., 2006).

\section{CONSIDERAÇÕES FINAIS}

Baseado nos resultados do presente estudo foi possível verificar associação entre o IMC, CC, CA, CQ, RCQ e a gordura corporal relativa quando analisados em voluntários com características físicas e antropométricas similares às do presente estudo.

Desta forma, observa-se a importância destes indicadores antropométricos, além da favorável relação com a gordura corporal relativa, fazendo com que estes indicadores se tornem uma ferramenta boa e confiável para o profissional de Educação Física, principalmente, com objetivo de estratificar a obesidade e relacionar a morbidade, principalmente, às doenças coronarianas.

\section{REFERÊNCIAS}

ACSM, 2014, American College of Sports Medicine; Pescatello LS, Arena R, Riebe D, Thompson PD, editors. ACSM's Guidelines for Exercise Testing and Prescription. 9th ed. Philadelphia (PA): Wolters Kluwer/Lippincott Williams \& Wilkins Health; 2014.

BRASIL. Ministério da Saúde. Secretaria de Vigilância em Saúde. VIGITEL. Brasil 2016: Hábitos dos brasileiros impactam no crescimento da obesidade e aumenta prevalência de diabetes e hipertensão. Brasília: Ministério da Saúde, 2016.

CASTRO, S. H; MATO, H. J; GOMES, M. B. Parâmetros antropométricos e síndrome metabólica em diabetes tipo 2. Arquivos Brasileiros de Endocrinologia \& Metabologia, v. 50, n. 3, p. 450-455, 2006. https://doi.org/10. 1590/ S0004-27302006000300007

CLASEY, J. L. et al. The use of anthropometric and dual-energy X-ray absorptiometry (DXA) measures to estimate total abdominal and abdominal visceral fat in men and women. Obesity, v. 7, n. 3, p. 256-264, 1999. https://doi.org/10.1002/j.1550-8528.1999.tb00404.x 
DA COSTA, V. C. et al. Fatores de risco cardiovascular em universitários de uma instituição de ensino superior privada. Revista Corpus Hippocraticum, v. 1, n. 1, 2017. https://doi.org/10.29148/labor.v1i18.31591

DEURENBERG, P; WESTSTRATE, J. A; SEIDELL, J. C. Body mass index as a measure of body fatness: age-and sex-specific prediction formulas. British Journal of Nutrition, v. 65, n. 2, p. 105-114, 1991. https://doi.org/10.1079/BJN19910073

FUJIOKA, S. et al. Contribution of intraabdominal fat accumulation to the impairment of glucose and lipid metabolism in human obesity. Metabolism, v. 36, n. 1, p. 5459, 1987. https://doi.org/10.1016/0026-0495(87) 90063-1

GARAULET, M. et al. Anthropometric, computed tomography and fat cell data in an obese population: relationship with insulin, leptin, tumor necrosis factor-alpha, sex hormonebinding globulin and sex hormones. European Journal of Endocrinology, v. 143, n. 5, p. 657-666, 2000. https://doi.org/10.1530/eje.0. 1430657

GONÇALVES, E. Equação de regressão com a perimetria e o dexa para a terceira idade. Rio de Janeiro: Castelo Branco, 2004.

GRINKER, J. A. et al. Changes in patterns of fatness in adult men in relation to serum indices of cardiovascular risk: the Normative Aging Study. International Journal of Obesity, v. 24, n. 10, p. 1369, 2000. https://doi.org/10.1038/sj.ijo.0801397

GROSSL, T; DE LIMA, L. R. A; KARASIAK, F. C. Relationship between percentage of body fat and anthropometric indicators in individuals attending a gym. Motricidade, v. 6, n. 2, p. 35-45, 2010. https://doi.org/10.6063/ motricidade.6(2).152

JACKSON, Andrew S.; POLLOCK, Michael L. Generalized equations for predicting body density of men. British Journal of Nutrition, v. 40, n. 3, p. 497-504, 1978. https://doi.org/10. 1079/BJN 19780152

KARTER, A. J. et al. Insulin sensitivity and abdominal obesity in African-American, Hispanic, and non-Hispanic white men and women: the Insulin Resistance and Atherosclerosis Study. Diabetes, v. 45, n. 11, p. $1547-$ 1555, 1996. https://doi.org/10.2337/diab.45.11. $\underline{1547}$

KEYS, A. et al. Indices of relative weight and obesity. International Journal of Epidemiology, v. 43, n. 3, p. 655-665, 2014. https://doi. org/10.1093/ije/dyu058

LEAN, M. E; HAN, T. S; DEURENBERG, P. Predicting body composition by densitometry from simple anthropometric measurements. The American Journal of Clinical Nutrition, v. 63, n. 1, p. 4-14, 1996. https://doi.org/10.1093/ajen/63.1.4

LEE, S. et al. Central obesity as a risk factor for prostatic hyperplasia. Obesity, v. 14, n. 1, p. 172-179, 2006. https://doi.org/10.1038/oby. $\underline{2006.21}$

MANGGE, H. et al. Inflammation, adiponectin, obesity and cardiovascular risk. Current Medicinal Chemistry, v. 17, n. 36, p. 4511 4520, 2010. https://doi.org/10.2174/092986710794 $\underline{183006}$

MARTI, A. et al. Genes, lifestyles and obesity. International Journal of Obesity, v. 28, p. S29-S36, 2004. https://doi.org/10.1038/sj.ijo. $\underline{0802808}$

MATERKO, W; SANTOS, E.L. Optimal cutoff values for obesity using classification tree in middle-aged adults living Rio de Janeiro city. International Journal of Research in Medical Sciences, v. 5, n. 7, p. 3172-3177, 2017. https://doi.org/10.18203/2320-6012.ijrms $\underline{20173008}$

MATERKO, Wollner et al. Accuracy of the WHO's body mass index cut-off points to measure gender-and age-specific obesity in middle-aged adults living in the city of Rio de Janeiro, Brazil. Journal of Public Health Research, v. 6, n. 2, 2017. https://doi.org/ 10.4081/iphr.2017.904

MATERKO, W; SANTOS, E.L. Predição e validação da gordura corporal relativa baseada em características antropométricas de adultos 
frequentadores de academia de ginástica. Arquivos em Movimento, v. 6, n. 1, p. 91-106, 2010. https://doi.org/10.6063/motricidade.6(2).152

OLIVEIRA, M. A. M. et al. Relação de indicadores antropométricos com fatores de risco para doença cardiovascular. Arquivos Brasileiros de Cardiologia, p. 478-485, 2010. https://doi.org/10.1590/S0066-782X2010005000012 OKOSUN, I. S. et al. Abdominal adiposity values associated with established body mass indexes in white, black and hispanic Americans. A study from the Third National Health and Nutrition Examination Survey. International journal of obesity, v. 24, n. 10, p. 1279, 2000. https://doi.org/10.1038/sj.ijo.0801414 PONGCHAIYAKUL, C. et al. Prediction of percentage body fat in rural Thai population using simple anthropometric measurements. Obesity, v. 13, n. 4, p. 729-738, 2005. https://doi.org/10.1038/oby.2005.82

POULIOT, MC et al. Waist circumference and abdominal sagittal diameter: best simple anthropometric indexes of abdominal visceral adipose tissue accumulation and related cardiovascular risk in men and women. The American Journal of Cardiology, v. 73, n. 7, p. 460-468, 1994. https://doi.org/10.1016/00029149(94)90676-9

REZENDE, F. A. C. et al. Índice de massa corporal e circunferência abdominal: associação com fatores de risco cardiovascular. Arquivos Brasileiros de Cardiologia, v. 87, n. 6, p. 728-34, 2006. https://doi.org/10.1590/ $\underline{\text { S0066-782X2006001900008 }}$

SARNO, F; MONTEIRO, C. A. Importância relativa do índice de massa corporal e da circunferência abdominal na predição da hipertensão arterial. Revista de Saúde Pública, v. 41, n. 5, p. 788-796, 2007. https://doi.org/10. 1590/S0034-89102007000500013

SCHREINER, P. J. et al. Sex-specific associations of magnetic resonance imaging-derived intra-abdominal and subcutaneous fat areas with conventional anthropometric indices:
The Atherosclerosis Risk in Communities Study. American Journal of Epidemiology, v. 144, n. 4, p. 335-345, 1996. https://doi.org/ 10.1093/oxfordjournals.aje.a008934

SIRI, W. E. Body composition from fluid spaces and density: analysis of methods. Techniques for measuring body composition, v. 61, p. 223-44, 1961.

STEWART, A. et al. International standards for anthropometric assessment. ISAK: International Society for the Advancement of Kinanthropometry. 2011.

WORLD HEALTH ORGANIZATION. Obesity: preventing and managing the global epidemic. World Health Organization, 2000.

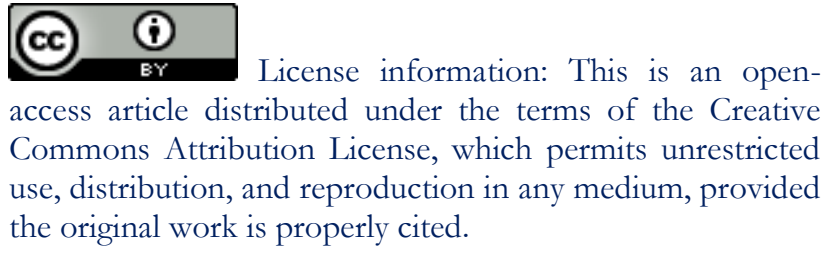

Artigo recebido em 18 de janeiro de 2018.

Avaliado em 05 de setembro de 2019.

Aceito em 25 de setembro de 2019.

Publicado em 02 de outubro de 2019.

\section{Como citar este artigo (ABNT):}

MATERKO, Wollner; COSTA JUNIOR, Paulo Jorge de Abreu da. Associação entre os indicadores antropométricos e a gordura corporal relativa em homens estudantes da Universidade Federal do Amapá, AP. Estação Científica (UNIFAP), Macapá, v. 9, n. 1, p. 31-37, jan./mar. 2019.0 